\title{
Outcomes of Redo Isolated Tricuspid Valve Surgery After Left-sided Valve Surgery
}

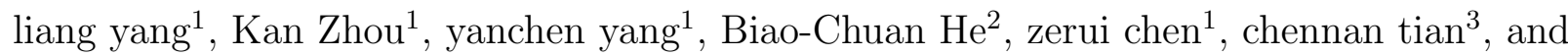 \\ huanlei huang ${ }^{3}$ \\ ${ }^{1}$ Guangdong Provincial People's Hospital \\ ${ }^{2}$ Guangdong Cardiovascular Institute \\ ${ }^{3}$ Affiliation not available
}

March 19, 2021

\begin{abstract}
Objectives: This study aimed to compare early and long-term outcomes of redo isolated tricuspid surgery (RITS) after left-sided valve surgery (LSVS). Methods: We retrospectively reviewed 173 patients underwent RITS for severe tricuspid regurgitation after previous LSVS from January 1999 to December 2019. Patients were divided into two groups: RITS by median sternotomy ( $\mathrm{m}$-RITS, $\mathrm{n}=78$ ) and by totally endoscopic approach (e-RITS, $\mathrm{n}=95$ ). Perioperative outcomes and follow-up results were analyzed. Results: There were $19(11 \%)$ in-hospital deaths (14.1\% in m-RITS and $8.4 \%$ in e-RITS, p $=0.234)$ that decreased from $16.7 \%(1999-2014)$ to $6.9 \%(2015-2019)(\mathrm{p}=0.044)$. Tricuspid valve replacement [odds ratio $(\mathrm{OR})=6.778,95 \%$ confidence interval (CI): $1.370-33.549, \mathrm{p}=0.019]$ and NYHA function class IV (OR $=8.525,95 \%$ CI: $2.153-33.760, \mathrm{p}=0.002)$ were independent risk factors of in-hospital mortality. The overall 1-, 5-year, 10-year, and 15-year survival rates were $97.2 \%(95 \%$ CI: $94.5-99.9 \%), 80.3 \%$ (95\% CI: $71.7-88.9 \%$ ), $59.2 \%$ (95\% CI: $43.5-75.5 \%$ ) and $49.3 \%$ (95\% CI: $27.2-71.4 \%$ ), respectively. Conclusion: Patients undergoing redo isolated tricuspid valve surgery carry a high risk of early mortality. Satisfactory results are achievable with endoscopic tricuspid valve surgery and repair results in lower surgical mortality than replacement with acceptable residual tricuspid regurgitation.
\end{abstract}

\section{Introduction}

Tricuspid regurgitation (TR) has become a substantial problem because of its high prevalence and poor clinical outcomes. A conservative estimate of total burden of moderate or severe TR in the US is 2.4 million residents both diagnosed and undiagnosed [1]. A large-scale investigation study in China indicated that the rates of moderate and severe TR was $2.22 \%$ and $1.39 \%$, respectively [2]. Long-term, higher-grade TR was an independent risk factor and associated with worse survival [3-5].

Clinical guidelines have adopted broader indications: surgery for mild-to-moderate TR with tricuspid annulus dilatation or right-heart failure undergoing left-sided valve surgery and primary symptomatic severe TR $[6$, 7]. Nevertheless, surgical correction and its optimal timing for isolated TR after left-sided valve surgery (LSVS) remains unclear.

Right mini-thoracotomy approach, beating-heart surgery, single venous drainage technique and other simplified surgical procedures have been recently applied in redo isolated tricuspid valve surgery (RITS), and outcomes of these evolving surgical strategies were encouraging [8-10]. The first totally endoscopic RITS after LSVS at our institution dated back to 2013; surgical techniques were later upgraded in 2015 [11-13], and postoperative and early outcomes were encouraging.

The present study aimed to review evolving strategies for RITS after LSVS at our institute in recent years, 
summarizing mid-to-long term outcomes and comparing the totally endoscopic approach with traditional median sternotomy approach.

\section{Materials and Methods}

The study protocol was approved by the Ethics Committee of Guangdong Provincial People's Hospital (GDREC2020177H), and the requirement for informed consent was waived because of the retrospective nature of the study.

\section{Study Population}

We retrospectively reviewed patients undergoing tricuspid valve surgery according to surgery ICD code (35.14; 35.28) from 1999 to 2019 in Guangdong Provincial Cardiovascular Institute. More than 15000 patients underwent tricuspid valve surgery in this period. The selection criteria were 1) previous LSVS with or without concomitant tricuspid valvuloplasty, and 2) RITS by an endoscopic approach or median sternotomy, tricuspid valve replacement (TVR) or tricuspid valve repair (TVr). The exclusion criteria were 1) TR due to endocarditis, congenital disease, trauma, or carcinoma, and 2) repeat TVR due to prostheses failure. A total of 173 cases were included and were divided into two subgroups: RITS by median sternotomy (m-RITS) and by totally endoscopic approach (e-RITS).

\section{Data Collection and Variable Definition}

Baseline information, operative and postoperative data were distracted from the inpatient records system. The examination results closest to surgery date were recorded. Low cardiac output syndrome was defined as supported by large doses of cardiovascular active drugs and mechanical equipment including intra-aortic balloon pump and extracorporeal membrane oxygenation. Respiratory complications were considered if any of the following conditions were met: 1) lung infection and pathogenic culture positive twice; 2) ventilation support more than 7 days when the heart function was good. Poor wound healing referred to the need for debridement.

\section{Tricuspid Valve Surgery and Evolving Strategies}

RITS carried a high risk and the decision to perform an operation was considerably cautious. The indications were previously described [11]. From 1998 to 2012, RITS were all performed through median sternotomy, and beating-heart or aortic cross-clamping were applied (Figure 1). The first case of e-RITS was performed late in 2013 and updated strategies of TVr with leaflets augmentation also started in 2015, and we preferred early RITS before New York Heart Association (NYHA) heart function class reached IV when patients had apparently impaired right heart function.

\section{Follow-up}

The follow-up was completed using out-patients visits, telephone calls, mail and online registration. Followup data consisted of death from any cause, redo tricuspid valve surgery, and heart transplantation. Overall survival was defined as the interval between operation date and the date of death.

\section{Statistical Analyses}

SPSS software, version 25.0 (SPSS Inc., Chicago, IL) was used for statistical analyses. If normally distributed, continuous variables were expressed as the mean \pm standard deviation and were analyzed using the Student's $t$ test. Non-normally distributed variables were expressed as median (first to third quartiles) and were compared using non-parametric tests. Categorical variables are presented as proportions and were compared using the chi-square test or Fisher's exact test. Univariate analysis was used to explore the relationship between perioperative data and follow-up outcomes. Variables with $P$-values less than 0.05 were entered into the multivariate analysis with logistic regression. Survival data were assessed using the Kaplan-Meier method and were compared using the log-rank test. AP -value less than 0.05 for all analyses was considered statistically significant.

\section{Results}




\section{Baseline Characteristics}

From January 1999 to December 2019, 173 (137 women, aged $54.87 \pm 8.77$ years) consecutive patients underwent RITS after previous left-sided valve surgery at our institute. Table 1 presents baseline characteristics of the 173 included patients. All the patients had previous cardiac procedures, including double valve replacement with or without concomitant $\mathrm{TVr}(\mathrm{n}=71)$, mitral valve replacement with or without concomitant $\mathrm{TVr}(\mathrm{n}=99)$, aortic valve replacement $(\mathrm{n}=1)$, mitral valve replacement concomitant with aortic valve repair and TVr $(\mathrm{n}=1)$, Bentall's surgery $(\mathrm{n}=1) ; 97.1 \%$ patients underwent LSVS for rheumatic heart valve disease. All patients had severe TR, and enlarged right atria and ventricles.

\section{Operative Data and Postoperative Outcomes}

Table 2 presents surgical data and postoperative outcomes for all the 173 patients. 101 patients underwent tricuspid valve replacement (TVR), including implantation of $83(58.4 \%)$ biological tricuspid valves and $18(10.4 \%)$ mechanical tricuspid valves; $72(41.6 \%)$ patients underwent TVr. The overall surgical volume increased in recent years, and the ratio of TVr increased from $9.0 \%$ to $76.2 \%$.

In-hospital deaths occurred in 19 patients for a mortality rate of $11.0 \%$. There was no significant difference between the m-RITS and e-RITS groups $(\mathrm{p}=0.234)$. The causes were heart and multi-organ failure $(\mathrm{n}=$ $12,63.2 \%)$, cardiac arrest $(\mathrm{n}=2,10.5 \%)$, infective endocarditis $(\mathrm{n}=1,5.3 \%)$, lung infection $(\mathrm{n}=2,10.5 \%)$, stroke $(\mathrm{n}=1,5.3 \%)$ and severe gastrointestinal bleeding $(\mathrm{n}=1,5.3 \%)$. The overall median cardiopulmonary bypass (CPB) time was 98.0 (75.0-118.5) minutes, and CPB time in the e-RITS group was longer than in the m-RITS group $(p<0.001)$. Patients in the e-RITS group had shorter intensive unit care stays and a lower proportion of reoperation for chest bleeding or poor wound healing. Univariable and multivariable logistic regression analyses indicated that replacement and NYHA function class IV were independent risk factors of in-hospital mortality (Table 3).

\section{Outcomes Stratified for Two Epochs}

Surgical strategies considerably changed since 2015, the application of leaflets augmentation increased the rate of TVr, and therefore we stratified patients into two epochs: 1999-2014 and 2015-2019 (Table 4). From 2015 to $2019,87.1 \%$ of patients underwent e-RITS and $62.4 \%$ underwent TVr. Before $2015,69.4 \%$ of patients underwent surgery with NYHA [?] III, while the ratio decreased to $45 \%$ in the recent 5 years $(p=0.002)$,

and only $12.5 \%$ underwent repair. In-hospital mortality also decreased from $16.7 \%$ to $6.9 \%(p=0.044)$ and fewer patients underwent reoperation due to chest bleeding or poor wound healing $(p=0.011$ and $p=0.021$, respectively)

\section{Follow-up Results}

Table 5, Figure 2 shows the details of follow-up results. The mean follow-up interval from operation date was 54.01 +- 44.37 months (ranged 3-241 months), and 150 (97.4\%) patients completed follow-up. A total of $26(16.5 \%)$ patients died during follow-up; the causes of death were cardiac related $(\mathrm{n}=21,80.8 \%)$, stroke $(\mathrm{n}=2,7.7 \%)$, cancer $(\mathrm{n}=1,3.8 \%)$, unclear $(\mathrm{n}=2,7.7 \%)$. The overall 1-, 5 -year, 10-year and 15-year survival rates were $97.2 \%$ [95\% confidence interval (CI): $94.5-99.9 \%$ ], $80.3 \%$ (95\% CI: $71.7-88.9 \%$ ), $59.2 \%$ (95\% CI: $43.5-75.5 \%)$ and $49.3 \%$ (95\% CI: $27.2-71.4 \%$ ), respectively. The 5-year survival rates were $85.2 \%$ (95\% CI: $62.1-94.1 \%)$ and $76.4 \%$ (95\% CI:65.6-87.2\%), respectively in the e-RITS and m-RITS groups, and no significant difference was found between groups $(p=0.122)$. Four patients underwent re-do TVR for protheses failures, one patient underwent re-do TVr for recurrent severe TR and two patients underwent transplantation for right heart failure.

\section{Discussion}

In this study, we introduced our singled-center experience of isolated tricuspid valve surgery in the past 20 years. We focused only on patients undergoing RITS after previous left-sided valve surgery, because we believe that this is high risk surgical population. Outcomes of this population might differ from those of patients undergoing first-time isolated tricuspid valve surgery and other cardiac procedures concomitant with 
redo tricuspid valve surgery. We reported early and long-term outcomes and evolving strategies applied in this population. The evolving strategies referred to innovated surgery techniques: early surgery if patients were considered candidates of surgery, totally endoscopic approach, and preferred $\mathrm{TVr}$ with leaflets augmentation when leaflet tethering was severe. The outcomes were encouraging, and both mortality and morbidity decreased since 2015 .

The volume of isolated tricuspid valve surgery has significant increased while still remaining relatively rare in comparison to 2.4 million residents with moderate to severe TR [1, 14]. During the study period, more than 15000 tricuspid valve surgeries were performed at our institute while RITS after LSVS accounted for only $1.1 \%$, and the procedures were rarely performed due to the relatively high in-hospital mortality ranging from $6 \%$ to $21 \%$ reported in previous reports [15-17]. However, we adopted a more active attitude towards surgery in the recent 5 years for the following reasons: 1) based on our clinical experiences, we found most patients sought surgery due to recurrent symptoms of right heart dysfunction with long-term medical treatment history and diuretics possibly being ineffective; 2) the totally endoscopic access combined with leaflet augmentation, simplified unicaval drainage without snare and beating-heart surgery techniques reduced ICU stay time and reoperation rate; and 3) we found TVr was effective and safe in patients with severe leaflet tethering [11].

Early studies introduced minimally invasive tricuspid valve surgery and reported lower mortality and morbidity; our evolving surgical techniques were different from those studies [10, 18]. More studies reported RITS after LSVS with a higher proportion of TVR due to leaflet structural alterations or excessive leaflet tethering in the context of a long-lasting disease when repair was difficult $[10,16,18]$. We prefer TVr, because TVR carries a higher risk of mortality and morbidity. Zack et al. reported their investigation of the nationwide trend of isolated tricuspid valve surgery and found higher in-hospital mortality for valve replacement than for repair, with a risk-adjusted odd ratio of 2.2 [14]. The present study also indicated that TVR was an independent risk factor with odds ratio (OR) of 6.778 (95\% CI: $1.370-33.549, p=0.019)$. The common issue [19] that tricuspid valve without coaptation and extensive annular dilation was no amenable to repair may persuade a surgeon to replace, while we believe that the augmentation of both anterior and posterior leaflet might address this issue by minimizing the leaflet tethering and increasing the coaptation. Therefore, we introduced the updated technique of leaflet patch augmentation of both anterior and posterior leaflets by sewing the patch to the leaflets and the annulus to convert the native leaflets into the coaptation zone and (partially) the chordae [13]. Other repair techniques such as ring implantation, artificial chordae, commissurotomy and papillary muscle incision were also performed as needed, and therefore the overall CPB time was long because of these combined repair techniques. Some surgeons might also be concerned about the increased rate of residual or recurrent TR and preferred TVR, our data showed acceptable results with $5.5 \%$ residual severe TR. These five patients with TVr had large preoperative jet area of TR with a median of $26.0(22.0-31.5) \mathrm{cm}^{2}$ that decreased to $13(11.5-14.0) \mathrm{cm}^{2}$ after surgery $(p=0.008)$.

The current controversies surrounding redo isolated tricuspid valve surgery are concentrated in the following aspects: 1) is surgery necessary and does surgery improve long-term outcomes of this population? 2) which surgical correction is better, repair or replacement? 3) when is the best time to consider surgery? Andrea et al. reported on 3276 patients from 2001 to 2016 and this large data set indicated that surgery did not improve survival of this population using a propensity-matched method [20]. Although patients with medical treatment were not included in the present series, our data indicated that surgical correction had excellent outcomes with 10-year survival rate of $59.2 \%$ (95\% CI: 43.5-75.5\%). Patients with repair had better early outcomes while there was no significant difference in the long-term survival. Another key finding was the increased mortality with higher NYHA function class. Patients with NYHA function class IV had relatively higher risk of in-hospital death with an OR of 8.525 (95\% CI: $2.153-33.760, p=0.002)$, and NYHA function class IV as an independent risk factor was frequently confirmed by previous studies [17, 18, $21,22]$. Considering the high risk of higher NYHA function class, we adopted early surgery strategies and preoperative optimized medical therapy are recommended to improve heart function.

\section{Limitations}


In addition to this being a single-center, retrospective study, there are several other limitations. The first is the wide heterogeneity of patient surgical data between the subgroups because of the variance in practice patterns from surgeon to surgeon and our team preferring to perform TVr using endoscopic approach. Second, objective assessments of tricuspid valve and right heart function are currently not available. Third, the updated surgery techniques began in 2015, the follow-up time was short. Long-term outcomes are needed to confirm its durability. Despite all these drawbacks, to our knowledge, the present study includes one of the largest population of patients undergoing RITS with mid-to-long term outcomes.

\section{Conclusions}

Patients undergoing redo isolated tricuspid valve surgery carry a high risk of early mortality. Satisfactory results are achievable in redo tricuspid valve surgery by endoscopic approach and repair results in lower surgical mortality than replacement with acceptable residual tricuspid regurgitation.

\section{Conflicts of Interest}

None.

\section{Funding}

This study was supported by Natural Science Foundation of Guangdong Province (2019B1515120071).

\section{Data Availability Statement}

Data are available from the corresponding author.

\section{Reference:}

1. Enriquez-Sarano M, Messika-Zeitoun D, Topilsky Y, et al. Tricuspid regurgitation is a public health crisis. Prog Cardiovasc Dis. 2019;62:447-451.

2. Yang L, Chen H, Pan W, et al. Analyses for Prevalence and Outcome of Tricuspid Regurgitation in China: An Echocardiography Study of 134,874 Patients. Cardiology. 2019;142:40-46.

3. Benfari G, Antoine C, Miller WL, et al. Excess Mortality Associated With Functional Tricuspid Regurgitation Complicating Heart Failure With Reduced Ejection Fraction. Circulation. 2019;140:196-206.

4. Topilsky Y, Inojosa JM, Benfari G, et al. Clinical presentation and outcome of tricuspid regurgitation in patients with systolic dysfunction. Eur Heart J. 2018;39:3584-3592.

5. Kazum SS, Sagie A, Shochat T, et al. Prevalence, Echocardiographic Correlations, and Clinical Outcome of Tricuspid Regurgitation in Patients with Significant Left Ventricular Dysfunction. Am J Med. 2019;132:8187.

6. Nishimura RA, Otto CM, Bonow RO, et al. 2014 AHA/ACC guideline for the management of patients with valvular heart disease: executive summary: a report of the American College of Cardiology/American Heart Association Task Force on Practice Guidelines. J Am Coll Cardiol. 2014;63:2438-2488.

7. Baumgartner H, Falk V, Bax JJ, et al. 2017 ESC/EACTS Guidelines for the management of valvular heart disease The Task Force for the Management of Valvular Heart Disease of the European Society of Cardiology (ESC) and the European Association for Cardio-Thoracic Surgery (EACTS). Eur Heart J. 2017;38:2739-2791.

8. Farber G, Tkebuchava S, Dawson RS, et al. Minimally Invasive, Isolated Tricuspid Valve Redo Surgery: A Safety and Outcome Analysis. Thorac Cardiovasc Surg. 2018;66:564-571.

9. Wang Q, Xue X, Yang J, et al. Right mini-thoracotomy approach reduces hospital stay and transfusion of mitral or tricuspid valve reoperation with non-inferior efficacy: evidence from propensity-matched study. J Thorac Dis. 2018;10:4789-4800.

10. Lu S, Song K, Yao W, et al. Simplified, minimally invasive, beating-heart technique for redo isolated tricuspid valve surgery. J Cardiothorac Surg. 2020;15:146. 
11. Chen Z, Ke Y, Xie X, et al. Outcomes of Totally Endoscopic Beating-Heart Tricuspid Repair in Redo Cardiac Surgery. Heart Lung Circ. 2020:1443-9506.

12. He BC, Ke YJ, Zhou K, et al. Modified unicaval drainage in reoperative isolated tricuspid valve repair via totally thoracoscopic approach. Perfusion. 2020;35:649-657.

13. Chen Z, Ke Y, Xie X, et al. Beating-Heart Totally Endoscopic Tricuspid Valvuloplasty in Reoperative Cardiac Surgery. Ann Thorac Surg. 2019;107:e79-e82.

14. Zack CJ, Fender EA, Chandrashekar P, et al. National Trends and Outcomes in Isolated Tricuspid Valve Surgery. J Am Coll Cardiol. 2017;70:2953-2960.

15. Bevan PJW, Haydock DA, Kang N. Long-term Survival after Isolated Tricuspid Valve Replacement. Heart Lung Circ. 2014;23:697-702.

16. Buzzatti N, Iaci G, Taramasso M, et al. Long-term outcomes of tricuspid valve replacement after previous left-side heart surgery. Eur J Cardiothorac Surg. 2014;46:713-719.

17. Oh TH, Wang TK, Sidhu K, et al. Isolated tricuspid valve surgery at a single centre: the 47-year Auckland experience, 1965-2011. Interact Cardiovasc Thorac Surg. 2014;18:27-32.

18. Chen J, Hu K, Ma W, et al. Isolated reoperation for tricuspid regurgitation after left-sided valve surgery: technique evolution. Eur J Cardiothorac Surg. 2020;57:142-150.

19. Bashore TM, Serfas JD. Isolated Tricuspid Valve Surgery. J Am Coll Cardiol. 2017;70:2961-2963.

20. Axtell AL, Bhambhani V, Moonsamy P, et al. Surgery Does Not Improve Survival in Patients With Isolated Severe Tricuspid Regurgitation. J Am Coll Cardiol. 2019;74:715-725.

21. Chen JM, Abudupataer M, Hu K, et al. Risk factors associated with perioperative morbidity and mortality following isolated tricuspid valve replacement. J Surg Res. 2018;221:224-231.

22. Topilsky Y, Khanna AD, Oh JK, et al. Preoperative factors associated with adverse outcome after tricuspid valve replacement. Circulation. 2011;123:1929-1939.

Table 1. Preoperative Data

\begin{tabular}{lllll}
\hline Variables & Overall $(\mathrm{n}=173)$ & $\mathrm{m}-\mathrm{RITS}(\mathrm{n}=78)$ & $\mathrm{e}-\mathrm{RITS}(\mathrm{n}=95)$ & $p$ va \\
\hline Female, $\mathrm{n}(\%)$ & $137(79.2)$ & $64(82.1)$ & $73(76.8)$ & 0.40 \\
Age, years & $54.87 \pm 8.77$ & $52.50 \pm 8.89$ & $56.81 \pm 8.22$ & 0.00 \\
Body mass index, $\mathrm{kg} / \mathrm{cm}^{2}$ & $22.49 \pm 2.98$ & $22.16 \pm 2.88$ & $22.76 \pm 3.05$ & 0.18 \\
Risk factors & & & & 0.03 \\
Hypertension, $\mathrm{n}(\%)$ & $35(20.2)$ & $10(12.8)$ & $25(26.3)$ & 0.09 \\
Diabetes mellitus, $\mathrm{n}(\%)$ & $13(7.5)$ & $3(3.8)$ & $10(10.5)$ & $>0.9$ \\
Previous rheumatic heart valve disease, $\mathrm{n}(\%)$ & $168(97.1)$ & $76(97.4)$ & $92(96.8)$ & 0.73 \\
Creatinine, umol/L & $64.0(56.0-79.9)$ & $64.0(56.9-82.0)$ & $64.0(55.6-77.3)$ & 0.73 \\
Albumin, g/L & $38.65 \pm 5.64$ & $38.81 \pm 6.50$ & $38.51 \pm 4.86$ & 0.73 \\
Atrial fibrillation/flutter, n (\%) & $168(97.1)$ & $75(96.2)$ & $93(97.9)$ & 0.40 \\
Pulmonary artery systolic pressure [?] $60 \mathrm{mmHg}, \mathrm{n}(\%)$ & $10(5.8)$ & $6(7.7)$ & $4(4.2)$ & 0.34 \\
NYHA class [?] III, n (\%) & $96(55.5)$ & $54(69.2)$ & $42(44.2)$ & 0.00 \\
NYHA class IV, n (\%) & $16(9.2)$ & $11(14.1)$ & $5(5.3)$ & 0.04 \\
Echocardiography & & & \\
RAD, mm & $82.24 \pm 17.78$ & $84.74 \pm 18.49$ & $80.19 \pm 17.01$ & 0.09 \\
RVED, mm & $60.48 \pm 8.57$ & $60.32 \pm 9.07$ & $60.61 \pm 8.19$ & 0.82 \\
LVEF, \% & $61.94 \pm 6.55$ & $61.70 \pm 6.63$ & $62.14 \pm 6.52$ & 0.66 \\
Systolic pulmonary artery pressure, $\mathrm{mmHg}$ & $41.0(36.0-48.0)$ & $41.0(36.0-48.0)$ & $40.0(35.0-48.0)$ & 0.56 \\
Jet area of tricuspid regurgitation, $\mathrm{cm}^{2}$ & $21.0(16.0-27.6)$ & $23.4(15.9-30.3)$ & $20.0(16.0-26.5)$ & 0.21 \\
& & & &
\end{tabular}




\begin{tabular}{|c|c|c|c|c|}
\hline Variables & Overall $(\mathrm{n}=173)$ & $\mathrm{m}-\operatorname{RITS}(\mathrm{n}=78)$ & $\mathrm{e}-\operatorname{RITS}(\mathrm{n}=95)$ & $p \mathrm{v}$ \\
\hline \multicolumn{5}{|l|}{ Previous cardiac surgery, n (\%) } \\
\hline DVR & $47(27.2)$ & $19(24.4)$ & $28(29.5)$ & 0.45 \\
\hline $\mathrm{DVR}+\mathrm{TVr}$ & $24(13.9)$ & $9(11.50$ & $15(15.8)$ & 0.4 \\
\hline MVR & $77(44.5)$ & $37(47.4)$ & $40(42.1)$ & 0. \\
\hline $\mathrm{MVR}+\mathrm{TVr}$ & $22(12.7)$ & $12(15.4)$ & $10(10.5)$ & 0. \\
\hline AVR & $1(0.6)$ & $0(0)$ & $1(1.1)$ & $>$ \\
\hline $\mathrm{MVR}+\mathrm{AVr}+\mathrm{TVr}$ & $1(0.6)$ & $1(1.3)$ & $0(0)$ & 0. \\
\hline Bentall's surgery & $1(0.6)$ & $0(0)$ & $1(1.1)$ & $>$ \\
\hline Interval from last heart operation, years & $14.0(10.0-20.0)$ & $11.0(8.8-17.0)$ & $16.0(11.0-22.0)$ & $<0$ \\
\hline
\end{tabular}

$\mathrm{AVR}=$ aortic valve replacement; $\mathrm{AVr}=$ aortic valve repair; $\mathrm{DVR}=$ double valve replacement; $\mathrm{e}-\mathrm{RITS}=$ endoscopic redo isolated tricuspid valve surgery; LVEF = left ventricular ejection fraction; $\mathrm{m}-\mathrm{RITS}=$ redo isolated tricuspid valve surgery by median sternotomy; MVR $=$ mitral valve replacement; NYHA $=$ New York Heart Association; RAD = right atrium diameter; RVED = right ventricular end-diastolic diameter;

Table 2. Operative Data and Post-operative Outcomes

\begin{tabular}{lllll}
\hline Variables & Overall $(\mathrm{n}=173)$ & $\mathrm{m}-$ RITS $(\mathrm{n}=78)$ & $\mathrm{e}-$ RITS $(\mathrm{n}=95)$ & $p$ value \\
\hline CPB time, minutes & $98.0(75.0-118.5)$ & $81.5(64.8-106.3)$ & $106(83.0-134.0)$ & $<0.001$ \\
Replacement, n (\%) & & & & \\
Bioprosthetic & $83(48.0)$ & $50(64.1)$ & $33(34.7)$ & $<0.001$ \\
Mechanical & $18(10.4)$ & $16(20.5)$ & $2(2.1)$ & $<0.001$ \\
Repair, n (\%) & $72(41.6)$ & $12(15.4)$ & $60(63.2)$ & $<0.001$ \\
In-hospital mortality, n (\%) & $19(11.0)$ & $11(14.1)$ & $8(8.4)$ & 0.234 \\
Ventilation time, hours & $21.5(12.8-30.5)$ & $26.0(19.0-46.0)$ & $19.0(10.0-26.0)$ & 0.103 \\
ICU stay, hours & $70.5(45.8-117.3)$ & $75.3(47.0-139.0)$ & $68(45.0-99.0)$ & $<0.001$ \\
Complication, n (\%) & & & & \\
Low cardiac output syndrome & $29(16.8)$ & $15(19.2)$ & $14(14.7)$ & 0.431 \\
Respiratory complication & $28(16.2)$ & $14(17.9)$ & $14(14.7)$ & 0.568 \\
Stroke & $2(1.2)$ & $1(1.3)$ & $1(1.1)$ & $>0.999$ \\
Dialysis & $19(11.0)$ & $9(11.5)$ & $10(10.5)$ & $>0.999$ \\
Reoperation for chest bleeding & $10(5.8)$ & $8(10.3)$ & $2(2.1)$ & 0.044 \\
Poor wound healing & $7(4.0)$ & $6(7.7)$ & $1(1.1)$ & 0.047 \\
\hline
\end{tabular}

$\mathrm{CPB}=$ cardiopulmonary bypass; - RITS $=$ endoscopic redo isolated tricuspid valve surgery; $\mathrm{m}-\mathrm{RITS}=$ redo isolated tricuspid valve surgery by median sternotomy; ICU = intensive care unit;

Table 3. Risk Factors for In-Hospital Mortality

\begin{tabular}{llll}
\hline Variable & Odds ratio & $95 \%$ confidence interval & $p$ value \\
\hline For univariate analysis & & & \\
Female & 0.306 & $0.113-0.829$ & 0.020 \\
Age & 1.071 & $1.006-1.14)$ & 0.033 \\
Body mass index & 1.074 & $0.920-1.254$ & 0.366 \\
Hypertension & 2.625 & $0.948-7.266$ & 0.063 \\
Diabetes mellitus & 1.529 & $0.312-7.487$ & 0.600 \\
Creatinine & 1.025 & $1.011-1.040$ & 0.001 \\
Albumin & 0.881 & $0.815-0.952$ & 0.001
\end{tabular}




\begin{tabular}{llll}
\hline Variable & Odds ratio & $95 \%$ confidence interval & $p$ value \\
\hline NYHA class [?] III & 7.962 & $1.778-35.650$ & 0.007 \\
NYHA class IV & 9.398 & $2.976-29.678$ & $<0.001$ \\
Interval from last heart operation & 0.979 & $0.910-1.053$ & 0.563 \\
RAD & 1.015 & $0.989-1.042$ & 0.261 \\
RVED & 1.048 & $0.993-1.105$ & 0.087 \\
LVEF & 1.012 & $0.941-1.089$ & 0.747 \\
Systolic pulmonary artery pressure & 1.040 & $1.000-1.082$ & 0.050 \\
Jet area & 1.040 & $1.000-1.081$ & 0.048 \\
Cardiopulmonary bypass time & 1.008 & $0.999-1.017$ & 0.066 \\
Median vs Endoscopic & 1.785 & $0.680-4.686$ & 0.239 \\
TVR vs TVr & 4.329 & $1.212-15.468$ & 0.024 \\
For multivariate analysis & & & \\
Female & 1.603 & $0.421-6.105$ & 0.489 \\
Age & 1.060 & $0.984-1.143$ & 0.126 \\
Creatinine & 1.020 & $0.984-1.143$ & 0.043 \\
Albumin & 0.888 & $0.805-0.979$ & 0.017 \\
NYHA class IV & 8.525 & $2.153-33.760$ & 0.002 \\
Jet area of TR & 1.009 & $0.961-1.061$ & 0.709 \\
TVR vs TVr & 6.778 & $1.370-33.549$ & 0.019 \\
\hline
\end{tabular}

NYHA $=$ New York Heart Association; RAD $=$ right atrium diameter; RVED $=$ right ventricular end diastolic diameter; $\mathrm{TVR}=$ tricuspid valve replacement; $\mathrm{TVr}=$ tricuspid valve repair;

Table 4. Data Stratified by Two Different Epochs.

\begin{tabular}{llll}
\hline Variables & Overall $(\mathrm{n}=173)$ & $1998-2014(\mathrm{n}=72)$ & $2015-2019(\mathrm{n}=101)$ \\
\hline Female, $\mathrm{n}(\%)$ & $137(79.2)$ & $58(80.6)$ & $79(78.2)$ \\
Age, years & $54.87 \pm 8.77$ & $52.56 \pm 8.71$ & $56.51 \pm 8.47$ \\
Risk factors & & & \\
Previous rheumatic heart valve disease, $\mathrm{n}(\%)$ & $168(97.1)$ & $70(97.2)$ & $98(97)$ \\
Atrial fibrillation/flutter, n (\%) & $168(97.1)$ & $70(97.2)$ & $98(97.0)$ \\
Pulmonary artery systolic pressure [?] 60 mmHg, $\mathrm{n}(\%)$ & $10(5.8)$ & $6(8.3)$ & $4(4.00$ \\
NYHA class [?] III, n (\%) & $96(55.5)$ & $50(69.4)$ & $46(45.5)$ \\
NYHA class IV, n (\%) & $16(9.2)$ & $11(15.3)$ & $5(5.0)$ \\
Interval from last heart operation, years & $14.0(10.0-20.0)$ & $11.0(8.0-15.0)$ & $16.0(12.0-22.0)$ \\
Surgical data & & & \\
Endoscopic access, $\mathrm{n}(\%)$ & $95(54.9)$ & $7(9.7)$ & $88(87.1)$ \\
Replacement, n (\%) & & & \\
Bioprosthetic & $83(48.0)$ & $47(65.2)$ & $36(35.6)$ \\
Mechanical & $18(10.4)$ & $16(22.2)$ & $2(2.0)$ \\
Repair, n (\%) & $72(41.6)$ & $9(12.5)$ & $63(62.4)$ \\
Cardiopulmonary bypass time, minutes & $98.0(75.0-118.5)$ & $80.0(64.0-101.5)$ & $107(83.0-132.0)$ \\
Ventilation time, hours & $21.5(12.8-30.5)$ & $24.5(19.0-46.0)$ & $20.5(10.0-26.0)$ \\
ICU stay, hours & $70.5(45.8-117.3)$ & $71.5(45.0-134.0)$ & $69.0(46.0-117.0)$ \\
Complication, n (\%) & & & \\
Low cardiac output syndrome & $29(16.8)$ & $16(22.2)$ & $13(12.9)$ \\
Respiratory complication & $28(16.2)$ & $14(19.4)$ & $14(13.9)$ \\
Reoperation for chest bleeding & $10(5.8)$ & $8(11.1)$ & $2(2.0)$ \\
Poor wound healing & $7(4.0)$ & $6(8.3)$ & $1(1.0)$ \\
& & &
\end{tabular}




\begin{tabular}{llll}
\hline Variables & Overall $(\mathrm{n}=173)$ & $1998-2014(\mathrm{n}=72)$ & $2015-2019(\mathrm{n}=101)$ \\
\hline In-hospital mortality, $\mathrm{n}(\%)$ & $19(11.0)$ & $12(16.7)$ & $7(6.9)$ \\
\hline
\end{tabular}

$\mathrm{ICU}=$ intensive care unit; NYHA = New York Heart Association;

Table 5. Follow-up Results

\begin{tabular}{llll}
\hline Variables & Overall $(\mathrm{n}=154)$ & $\mathrm{m}-$ RITS $(\mathrm{n}=67)$ & $\mathrm{e}-$ RITS $(\mathrm{n}=87)$ \\
\hline Median follow-up time, months & $53.83 \pm 44.57$ & $85.44 \pm 51.36$ & $31.30 \pm 17.71$ \\
Follow-up completed, n (\%) & $150(97.4)$ & $64(95.5)$ & $86(98.9)$ \\
Death during follow-up, n (\%) & $26(16.9)$ & $22(32.8)$ & $4(4.6)$ \\
NYHA heart classification & $1.77(1.00-2.00)$ & $1.82(1.00-2.00)$ & $1.76(1.00-2.00)$ \\
Reoperation, n (\%) & & $4(5.8)$ & $1(1.1)$ \\
Redo tricuspid valve surgery & $5(3.2)$ & $2(3.0)$ & $0(0)$ \\
Heart transplantation & $2(1.3)$ &
\end{tabular}

e-RITS = endoscopic redo isolated tricuspid valve surgery; m-RITS = redo isolated tricuspid valve surgery by median sternotomy; NYHA = New York Heart Association;

\section{Figure Legends}

Figure 1: Increasing surgical volume and evolving technique at our institution from January 1999 to December 2019. e-RITS = endoscopic redo isolated tricuspid valve surgery; $\mathrm{m}-\mathrm{RITS}=$ redo isolated tricuspid valve surgery by median sternotomy; TVR = tricuspid valve replacement; $\mathrm{TVr}=$ tricuspid valve repair.

Figure 2: Kaplan-Meier curves of Survival for all patients and comparison between different groups. eRITS $=$ endoscopic redo isolated tricuspid valve surgery; $\mathrm{m}-$ RITS $=$ redo isolated tricuspid valve surgery by median sternotomy; TVR = tricuspid valve replacement; $\mathrm{TVr}=$ tricuspid valve repair;MTV = mechanical tricuspid valve; $\mathrm{BTV}=$ biological tricuspid valve. 

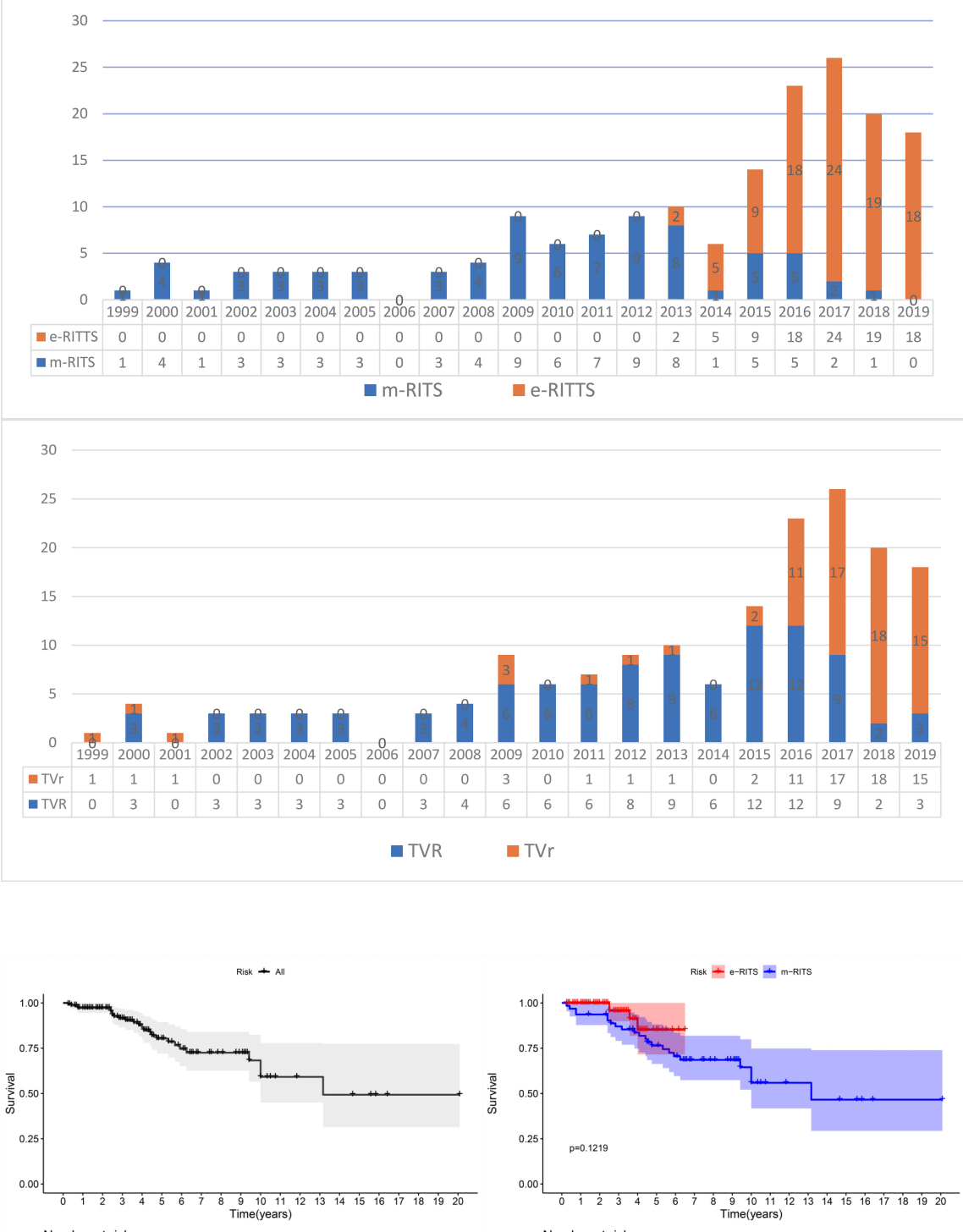

Number at risk

Number at risk

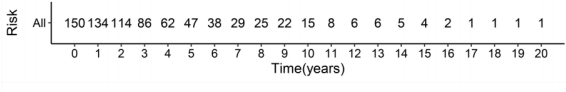

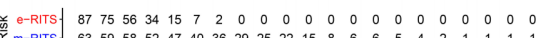
Risk $=\mathrm{TVr}+\mathrm{TVR}$
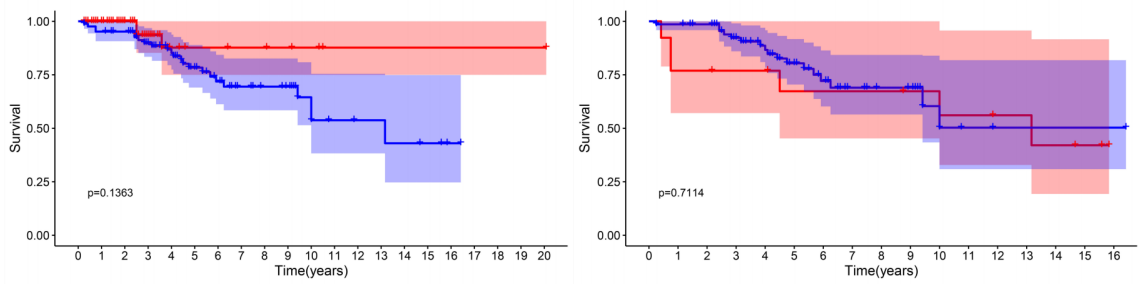
Number at risk Number at risk

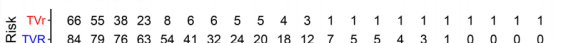

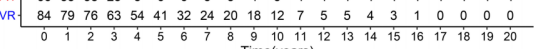

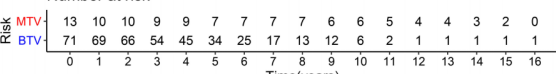

